\title{
Harmonic Balance algorithm to model nonlinear effects in HTS filters subject to CDMA signals
}

\author{
J. Mateu, C. Collado, J. M. O'Callaghan
}

\begin{abstract}
We discuss an Harmonic Balance algorithm to analyze High Temperature Superconducting Circuits (HTS) subject to wideband signals, such as the ones used in CDMA wireless systems. Undersampling is used to discretize and down-convert the bandpass signal resulting from the weak HTS nonlinearities, and this has to be taken into account when performing time-domain calculations of the nonlinear effects. We present an overview of the algorithm and describe an example to verify the software developed. We also discuss a tentative performance assessment of an HTS preselect filter in a UMTS base station receiver.
\end{abstract}

Keywords- CDMA, harmonic balance, HTS, Intermodulation, Nonlinearities, superconducting filters.

\section{Introduction}

Many second and third generation wireless systems use CDMA spread spectrum techniques to encode voice and data traffic and multiplex users onto the same frequency channel. Important enhancements in system performance have been obtained by using cryogenic front-ends in the base stations of these systems [1]. These front-ends include High Temperature Superconducting (HTS) pre-select filters and cooled low noise amplifiers to achieve superior sensitivity and frequency selectivity, which should make them immune to interfering signals from other operators and services. However, there is concern on the possible degradation brought by the microwave nonlinearities of the HTS materials.

HTS pre-select filters are usually made in microstrip technology and, since their nonlinear behavior is due to the properties of the HTS, the nonlinearities are distributed along the circuit pattern [2].This is unlike most other microwave circuits whose nonlinearities are due to lumped components such as diodes and transistors. Calculation of the effects of HTS nonlinearities often requires specialized software to account for their distributed nature, and to combine it with the convoluted layouts that resonators in HTS filters usually have (see, for example [3]).

Many previous works have studied how HTS nonlinearities produce two-tone intermodulation in filters and resonators $[4],[5]$. Among them, we have worked on computer codes based on Harmonic Balance (HB) [6] that combine models of the local nonlinearities of HTS materials with

This work is supported by the Spanish Ministry of Science and Technology through project TIC2000-0996 and scholarship AP9978085980 , and by Generalitat de Catalunya (DURSI) through grant 2001 SGR 0026.

J. Mateu is with the Telecommunication Technological Center of Catalonia (CTTC). Ed. Nexus. C/ Jordi Girona 1. Barcelona, Spain 08034

C. Collado and J.M O'Callaghan are with Universitat Politècnica de Catalunya (UPC), Campus Nord UPC D3. C/ Jordi Girona 1. Barcelona, Spain 08034 (email: joano@tsc.upc.es).

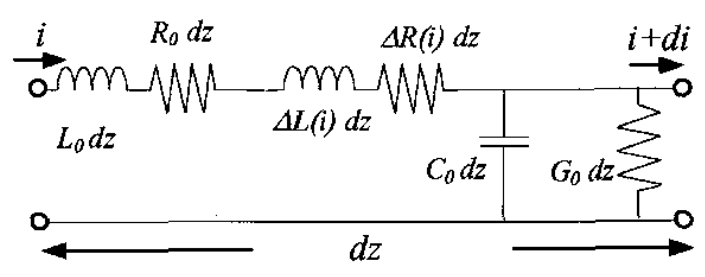

Fig. 1. Equivalent circuit valid for a small segment of HTS nonlinear transmission line of length $\mathrm{dz}$, or for a small annular region of an HTS disk resonator with width $\mathrm{dz}$.

circuit or electromagnetic models that account for the fact that nonlinearities are distributed [7], [8]. However, there is still very little work on how HTS devices respond to CDMA signals. Here we attempt to we bridge this gap by presenting how to modify our previous algorithms to simulate the nonlinear effects produced by driving HTS devices with wideband signals such as the ones used in spread spectrum wireless systems. We refer to the resulting algorithm as Multiport Multitone Harmonic Balance (MMHB) to point out the large number of frequencies and nonlinear elements considered.

\section{Multitone Multiport Harmonic Balance}

Our algorithms discretize all the distributed resonators in a filter to account for spatially distributed nonlinearities. For the case of transmission line filters, this discretization consists in cascading many identical two-ports, each one modelling a segment of the transmission line much smaller than a wavelength (Fig. 1). This type of discretization is also used for modelling nonlinear effects in disk resonator filters [9]. These type of resonators are used in HTS filters when high power handling is required, since they can hold azimuthally symmetric modes having field configurations that do not generate large current densities in the HTS. Thus, the circuit in Fig. 1 can also model a small annular region of a disk resonator.

In this work we will focus on these resonators i.e., those that can be modelled as a cascade of two-ports like the one in Fig. 1. Modelling a filter with several resonators results in a large nonlinear circuit (Fig. 2), which we analyze using numerical methods based on $\mathrm{HB}$ [6]. In our case, the nonlinear part of the circuit would model the nonlinearities in the resistance and inductance per unit length of the transmission line or disk resonators (Fig. 1), and the linear network would model all other (linear) elements in the circuit (the linear resistance, inductance, capacitance 


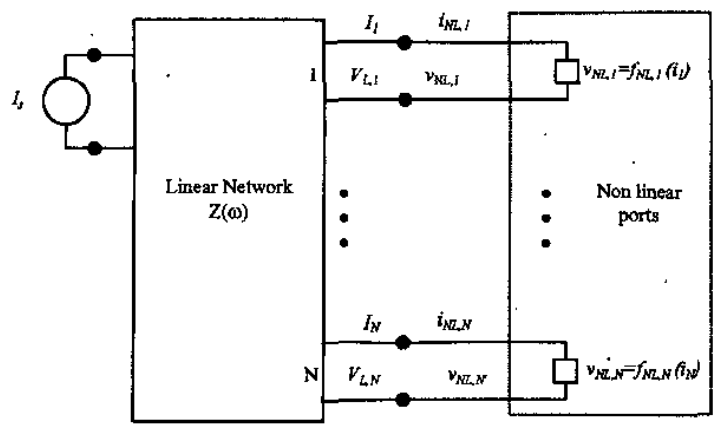

Fig. 2. Circuit to be analyzed using Harmonic Banance. There are $\mathrm{N}$ non-linear one-ports coming from the discretization of transmission lines or disk resonators. The linear $\mathrm{N}+1$ port contains all the linear filter elements, including coupling between resonators, coupling to the external source, and the output load and ita coupling.

and conductance per unit length of the line, the coupling between lines, etc.) [7].

The voltage $v_{N L}$ across the $\mathrm{N}$ nonlinear one-ports in Fig. 2 depends on the current through them $\left(i_{N L}\right)$ by means of a nonlinear resistance $\left(\Delta R\left(i_{N L}\right)\right)$ and inductance $\left(\Delta L\left(i_{N L}\right)\right)$. This voltage is calculated in time domain, i.e. [4]:

$$
v_{N L}\left(i_{N L}\right)=\Delta R\left(\dot{i}_{N L}\right) i_{N L}+\frac{d}{d t}\left[\Delta L\left(i_{N L}\right) i_{N L}\right] .
$$

The linear $(\mathrm{N}+1)$-port in Fig. 2 is characterized in frequency domain by an impedance matrix (Z). In principle, the values of the matrix elements have to be calculated at all frequencies of the source current, and at all other frequencies where spurious signals may exist.

\section{A. Undersampling and down-conversion of nonlinear spec- tra}

Our procedure starts by approximating the spectrum of a wideband signal by a set of closely-spaced spectral lines close to a carrier frequency $f_{0}$ in steps of $\Delta f$ ranging from $f_{0}-l \Delta f$ to $f_{0}+k \Delta f:$

$$
s(t) \approx \operatorname{Re}\left\{\sum_{i=-l}^{i=k} a_{i} e^{j 2 \pi\left(f_{0}+i \Delta f\right) t}\right\}
$$

where $a_{i}$ is the complex amplitude of each spectral line, and $k, l$ are integers. The frequency resolution $\Delta f$ is much smaller than $f_{0}$ and is fixed by the duration of the time window taken for the analysis of the signal $(1 / \Delta f)$. If this signal is subject to nonlinearities, it will result in another signal $y(t)$ with a larger number of closely spaced spectral lines grouped at odd multiples of the center frequency $\left(f_{0}\right.$, $\left.3 f_{0}, 5 f_{0} \ldots\right)$. Since nonlinearities are weak, we can neglect the spectral components about $5 f_{0}$ and higher and $y(t)$ can be written as:

$$
y(t)=\operatorname{Re}\left\{\sum_{\kappa_{\mathrm{qn}}} b_{q_{\mathrm{n}}} e^{j 2 \pi \kappa_{\mathrm{qn}} t}\right\}
$$

with $\kappa_{q, n}=v_{q, n}+n f_{0}$ with $n=1,3$ and $v_{q, n}$ are the frequencies of the spectral lines with respect to the multiples of $f_{0}$, that is $[10]$ :

$$
\begin{gathered}
\kappa_{q, n}=\left[f_{0}-(2 l+k) \Delta f, \cdots, f_{0}-\Delta f, f_{0}\right. \\
, f_{0}+\Delta f, \cdots, f_{0}+(2 k+l) \Delta f \\
3\left(f_{0}-l \Delta f\right), \cdots, 3 f_{0}-3 \Delta f, 3 f_{0} \\
\left.3 f_{0}+3 \Delta f, \cdots, 3\left(f_{0}+k \Delta f\right)\right] .
\end{gathered}
$$

Sampling the spectrum of $y(t)$ using standard techniques would require a sampling rate of at least twice its maximum frequency, i.e. $6\left(f_{0}+k \Delta f\right)$, rosulting in an unpractically large number of samples. However, since the signal is bandlimited, it is possible to downshift the signal to lower frequencies without lasing information by choosing a sampling rate $f_{s}$ much lower than $f_{0}$. An analysis on how sampling $y(t)$ at $f_{s}$ affects the resulting spectrum shows that a downconverted copy of the original spectrum around $f_{0}$ appears at a much lower frequency $f_{0}^{\prime}$ that satisfies $f_{0}^{\prime}=f_{0}-p f_{s}$, where $p$ is an integer such that $p f_{s}<f_{0}<(p+1) f_{s}$. Also, a down-converted copy of the spectrum around $3 f_{0}$ appears at $3 f_{0}^{\prime}$. To avoid aliasing in these two down-converted spectra, the following conditions have to be satisfied:

$$
\begin{gathered}
f_{0}^{\prime}>(2 l+k) \Delta f \\
f_{s} / 2>3\left(f_{0}^{\prime}+k \Delta f\right) .
\end{gathered}
$$

Thus, to determine the sampling rate $\left(f_{s}\right)$, we first find $f_{0}^{\prime}$ according to (5) and then find the value of $f_{s}$ that satisfies (6) and makes $\left(f_{0}-f_{0}^{\prime}\right) / f_{s}$ integer, so that the conditions $f_{0}^{\prime}=f_{0}-p f_{s}$ and $p f_{s}<f_{0}<(p+1) f_{s}$ are also fulfilled. Since CDMA signals do not have sharp spectral decays, ample margin are given to these conditions.

The down-converted version of $y(t)\left(y_{l}(t)\right)$ can be written as

$$
y_{l}(t)=\operatorname{Re}\left\{\sum_{d_{\mathrm{qn}}} b_{q_{\mathrm{n}}} e^{j 2 \pi d_{\mathrm{q}, \mathrm{n}^{t}}}\right\}
$$

where $d_{q, n}=v_{q_{n}}+n f_{0}^{\prime}$, i.e. the frequencies in (7) can be obtained from those listed in (4) by replacing $f_{0}$ by $f_{0}^{\prime}$.

\section{B. Derivatives and down-converted signals}

A central feature of the Harmonic Balance algorithm is the calculation of nonlinearities in time domain. In our case (see (1)), this calculation includes reactive nonlinearities whose calculation involves taking derivatives. Even though we work with down-converted signals, these derivatives should be done with the original signals which have a much faster time variations than the down-converted ones. In other words, if $y(t)$ represents the time-domain signal $\Delta L\left(i_{N L}\right) i_{N L}$ in (1) and we are working with its downconverted signal $y_{l}(t)$ in (7), doing the nonlinear calculation in (1) involves calculating the down-conversion of $d y / d t$, i.e.:

$$
\left.\frac{d y(t)}{d t}\right|_{l}=\operatorname{Re}\left\{\sum_{d_{q n}} j 2 \pi \kappa_{q n} b_{q_{\mathrm{n}}} e^{j 2 \pi d_{q n} t}\right\} .
$$

Note that this produces a different result than taking the derivative of $y_{l}(t)$. 


\section{Impedance matrix size}

One of the most restrictive limitations of MMHB is the size of the matrix $Z$, which depends on the number of nonlinear cells and the number of frequency components being considered [4]. Strictly speaking, Z should be defined at all frequencies of the signal source, and at the frequencies of all the spurious produced by the device, i.e. the frequencies listed in (4). However, for the case of a filter, only the components around $f_{0}$ fall within the filter passband and therefore, $Z$ only needs to be calculated at these frequencies $\left(f_{0}-(2 l+k) \Delta f, \ldots, f_{0}, \ldots, f_{0}+(2 k+l) \Delta f\right)$. In other words, the spectral components of the voltages across the nonlinear one-ports whose frequencies are significantly different from $f_{0}$ will not launch strong resonant fields in the filter that can affect the signals that we are interested in (those around $f_{0}$ ), and thus they can be ignored. Note that this does not change the restrictions on the sampling rate described in Sect. II-A

\section{MMHB outline}

The preceding discussions result in the following outline of the MMHB method:

1. Determination of the frequency resolution $\Delta f$ needed.

2. Determination of frequencies $\kappa_{q n}$ : Once $\Delta f$ is decided, $k$ and $l$ are determined to fit the bandwidth of the signal and the frequencies of the signals to be considered $\left(\kappa_{q n}\right)$ are calculated using (4).

3. Calculation of the matrix $Z$ at $f_{0}-(2 l+k) \Delta f, \ldots, f_{0}, \ldots, f_{0}+$ $(2 k+l) \Delta f$.

4. Determination of the center frequency of the downconverted signals $f_{0}^{\prime}$ and sampling rate $f_{s}$.

5. Determination of the frequencies of the down-converted signals $d_{q n}$.

6. Start the Harmonic Balance iterative algorithm (see Fig. 3 of [4] and Fig. 2):

(a) Propose a solution for the current through the $\mathrm{N}$ nonlinear one-ports $i_{N L}$. The initial estimate is calculated from the circuit in Fig. 2 assuming that the voltage across the nonlinear one-ports is zero.

(b) With the down-converted version of $i_{N L}$, find the voltage across the nonlinear one-ports $v_{N L}$ by applying (1), (7) and (8).

(c) Transform $v_{N L}$ to frequency domain and find its upconverted counterpart by moving its frequency components from $d_{q n}$ to $\kappa_{q n}$.

(d) With the voltage resulting from the previous point, the source current $I_{s}$, and the matrix $\mathrm{Z}$, solve the linear circuit and find an updated estimate of the current thought the one-ports $I_{N L}$.

(e) Down-convert $I_{N L}$ and transform it to time domain. Compare it with $i_{N L}$ in point (b) above. If convergence is not achieved go to point (b) and start a new iteration.

\section{E. Verification}

To verify the algorithm, we analyze the intermodulation products generated by. a HTS half-wave microstrip resonator fed by two tones, both of which are within the bandpass of the resonator. In this type of resonator, the nonlinearities in the HTS give rise to a current dependent inductance and resistance per unit length in the line $(\Delta L(i), \Delta R(i)$, see Fig. 1). We have developed equations that relate $\Delta L(i), \Delta R(i)$ with the amplitude of the intermodulation products in the resonator [11]. To verify the software developed, we have compared the result of these equations with the output of our software for the values of $\Delta L(i), \Delta R(i)$ of two different HTS samples supplied by established vendors [12] (see Annex). Note that, even though our algorithm is developed for wideband signals, it can also handle narrowband ones, like those of this verification example.

Table I shows the results of the comparison between theoretical and computed values for a two-port resonator $(28.5$ $\mathrm{mm}$ long ) with a resonant frequency of $1.94 \mathrm{GHz}$, an available power of $-30 \mathrm{dBm}$ per tone, a frequency separation between tones of $\Delta f=10 \mathrm{~Hz}$, and a coupling coefficient of $\beta=4.55$. The results in this table are given in terms of the peak intermodulation current at the center of the resonator, and the agreement between simulation and theory is within $0.5 \%$.

\begin{tabular}{|l|l|l|}
\hline & $H T S_{1}$ & $H_{T S}$ \\
\hline$I_{I M D_{\operatorname{sim}}(\mu A)}$ & 31.84 & 3.85 \\
\hline Error $(\%)$ & $<0.2$ & $<0.5$ \\
\hline
\end{tabular}

TABLE I

Peak intermodulation current

\section{Performance of a HTS pre-select filter in a UMTS base station}

The software developed can help predict the nonlinear performance of HTS filters in base stations of wireless systems using CDMA signals. In this first tentative assessment, we have assumed an 8 th order quasi-elliptic filter, similar to the one described in [13]. We have modelled the resonators as straight half-wave lines, such as the one in the verification example above. We thing this might not introduce significant differences with respect to some of the resonators used in HTS filters, such as the open-loop resonator, in which the current distribution in the parts of the line close to the current maximum resembles closely that of the straight half-wave resonator.

In our equivalent circuit, couplings between resonators are modelled with ideal impedance inverters, whose values are adjusted to obtain the desired passband response over a bandwidth of $15 \mathrm{Mhz}$ at a central frequency of $1.9425 \mathrm{GHz}$ (3 FDD UMTS uplink channels, see Fig. 3).

We have simulated a near-far scenario [14], where an ideal mobile terminal is transmitting at maximum power close to a base station of a wireless operator with an adjacent spectrum allocation (Fig. 3). We also assume that this base station is receiving $-120 \mathrm{dBm}$ signals from three users, each one in a $5 \mathrm{MHz}$ bandwidth, filling the $15 \mathrm{MHz}$ passband of the base station receiver. According to the 3GPP specifications, the interfering terminal may emit at $21 \mathrm{dBm}$ output power, and the minimum path loss to the 


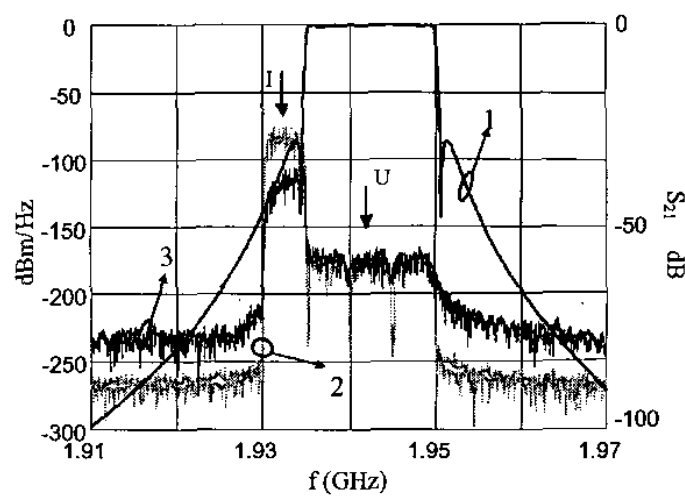

Fig. 3. Interference scenario in a UMTS FDD uplink pre-select filter with HTS materials: (1) Linear frequency response of the filter; (2) Input power spectral density; (3) Output power spectral density; (I) interferering channel; (U) User channel being considered.

base station is $50 \mathrm{~dB}$, so the interfering power is $-29 \mathrm{dBm}$. The nonlinear effects in Fig. 3 have been calculated with sample $H T S_{1}$, which is the one showing stronger intermodulation distortion in Table I. The equivalent results using sample $H T S_{2}$ show two effects:

1. Unlike in Fig. 3, the output power outside the passband is significantly below the input power.

2. The power leakage from the interferer (I) to the $5 \mathrm{MHz}$ user channel in the center of de passband (U) (see Fig. 3) is reduced by $3.8 \mathrm{~dB}$.

Both effects are due to the spectral regrowth caused by the stronger nonlinearities in sample $H T S_{1}$, which are noticeable despite the low power levels involved, and may cause a degradation in the traffic capacity of the base station.

Similar effects can be predicted for interferences caused by the UMTS TDD signals on this type of receiver (UMTS FDD).

\section{Conclusions}

We have described the basics of the extension of our Harmonic Balance algorithms to allow them to analyze the effects of signals with many frequency components on High Temperature Superconductors. This capability is of interest since it allows to evaluate the effect of superconductor nonlinearities in filters subject to CDMA signals. The extended algorithm (MMHB) compares well with the previous ones when analyzing two-tone intermodulation, and is producing reasonable results when predicting the performance of HTS filters with 3G CDMA signals. A tentative performance assessment of a pre-select filter in a UMTS base station shows that sample quality might affect the performance of the base station, despite the low powers involved. This might raise the need for screening the nonlinear properties of HTS samples prior to filter fabrication.

\section{Annex: HTS microstrip nonlinearities}

We assume that the HrS material used in the res onator is YBCO as the one supplied by established vendors $\left(R_{s}=20 \mu \Omega\right.$ at $\left.77 \mathrm{~K}\right)$. We have recently studied the nonlinearities of these type of samples [12] and found that the intermodulation properties can change significantly from sample to sample. Applying the results of [12] to a $1 \mathrm{~mm}$ wide microstrip line over a $508 \mu \mathrm{m}$ thick MgO dielectric $\left(\epsilon_{r}=9.8, \tan \delta=2 \cdot 10^{-6}\right)$, we can predict the dependence of the inductance and resistance per unit length on the current through it and thus, the values of $\Delta R(i)$ and $\Delta L(i)$ of Fig. 1. For sample $H T S_{1}$, this results in $\Delta L(i)=$ $3.8 \times 10^{-12}|i|^{0.2}$ and $\Delta R(i)=2.65 \times 10^{-3}|i|^{0.2}$, whereas for $H T S_{2}, \Delta L(i)=2.2 \times 10^{-12}|i|$ and $\Delta R(i)=2.2 \times 10^{-12}|i|$ (in all cases $i$ is in $\mathrm{A}, \Delta L$ is in $\mathrm{H}$ and $\Delta R$ in $\Omega$ ).

\section{References}

[1] B. A. Willemsen, "HTS Filter Subsystems for Wireless Telecommunications", IEEE Trans. Applied Superconductivity 11(1), p.60-67 (2001)

[2] T. Dahm and D. J. Scalapino, "Theory of intermodulation in a superconductiong microstrip resonator", Journal of Applied Physics 81, 2002-2009 (1997).

[3] E. R. Soares, "Design and contruction of High performance HTS pseudo-elliptic bandstop filter", IEEE MTT-S Digest (1999).

[4] C.Collado, J. Mateu, and J. O'Callaghan, "Harmonic Balance Algorithms for the Nonlinear Simulation of HTS Devices", Journał Applied Superconductivity , p. 57-64 (2001).

[5] D. Oates, "Nonlinear Behavior of Superconducting devices", in Microwave Superconductivity, edited by $\mathrm{H}$. Weinstock and M. Nisenoff, volume 375 , page p. 117, Air Force Office of Scientific Research, 2001.

[6] S. A. Maas, Nonlinear Microwave Circuits, Artech House, 1988. C.Collado, J. Mateu, and J. O'Callaghan, "Nonlinear Simulation and Characterization of HTS Devices using Harmonic Balance Algorithm", IEEE. Trans. On Applied superconductivity 11(1), p. 1396-1399 (2001).

[8] J. Parron, C.collado, J. Mateu, and J. O'Callaghan, "General Electromagnetic Simulation Tool to Predict the Microwave Nonlinear Response of Planar, Arbitrarily-Shaped HTS structures", IEEE. Trans. On Applied superconductivity 11(1), p. 399-402 (2001).

[9] J. Mateu, C.Collado, and J. O'Callaghan, "Nonlinear Analysis of Disk Resonators. Application to Material Characteritzation and Filter Desing", IEEE. Trans. On Applied superconductivity $11(1)$, p. 135-138 (2001).

[10] V. Borich, J. East, and G. Haddad, "An Efficient Fourier Transform for Multitone Harmonic Balance", IEEE Trans. Microwave Thery Techniques 47(2), p. 182 (1999).

[11] J. Mateu, Nonlinear distortion in HTS microwave devices and systems. PhD Thesis., Barcelona, UPC, 2003.

[12] J. Mateu, C.Collado, O. Menendez, and J. O'Callaghan, "A general approach for the calculation of intermodulation distortion in cavities with superconducting endplates", Applied Physics Letters in press.

[13] J.-S. Hong, M. Lancaster, D. Jedamzik, and R. B. Greed, "On the Development of Superconducting Microstrip Filters for Mobile Communications Applications", IEEE Trans. On Microwave Theory and Techniques $\mathbf{4 7}(9)$, p. 1656 (1999).

[14] H. Holma and A. Taskala, WCDMA for UMTS, Wiley, 2000. 